Case Report

\title{
Concurrent Polycythemia of Undetermined Etiology and Smouldering Plasma Cell Myeloma
}

\author{
Roula Katerji $(\mathbb{D})$ and Chad A. Hudson $(\mathbb{D}$ \\ Department of Pathology and Laboratory Medicine, University of Rochester, Rochester, NY, USA \\ Correspondence should be addressed to Roula Katerji; roula_katerji@urmc.rochester.edu
}

Received 10 July 2018; Accepted 6 August 2018; Published 16 September 2018

Academic Editor: Achille Pich

Copyright (C) 2018 Roula Katerji and Chad A. Hudson. This is an open access article distributed under the Creative Commons Attribution License, which permits unrestricted use, distribution, and reproduction in any medium, provided the original work is properly cited.

\begin{abstract}
The combination of polycythemia and plasma cell myeloma occurring concurrently is very rare and few cases have been reported in the literature. Further, the vast majority of these cases are cases of polycythemia vera and myeloma. Here, we present a case of polycythemia of undetermined etiology and myeloma. The patient is a 48-year-old Caucasian male who was originally diagnosed with polycythemia of undetermined etiology. Twelve years later, when a bone marrow biopsy was performed in an attempt to determine the etiology of the polycythemia, findings diagnostic of plasma cell myeloma were discovered. Subsequent serum studies were also consistent with a plasma cell neoplasm, while evaluation for end-organ damage was negative. A battery of genetic and biochemical tests ruled out various congenital polycythemias, leading to a final diagnosis of polycythemia of undetermined etiology and smouldering plasma cell myeloma. This case highlights that while being unusual, polycythemia and plasma cell myeloma can occur concurrently, and, in this report, we discuss both entities and potential mechanisms of the pathophysiology of the concurrent presentation.
\end{abstract}

\section{Introduction}

Plasma cell myeloma (PCM) accounts for approximately $1.6 \%$ of all cancer cases and about $10 \%$ of all hematologic malignancies in the United States [1] and is characterized by the proliferation of malignant plasma cells and subsequent increase of a monoclonal paraprotein in serum. Studies usually done when suspecting myeloma include serum and urine electrophoresis, serum-free light chain assay, bone marrow aspiration and biopsy with karyotype and FISH analysis, measurement of serum levels of beta-2 microglobulin, albumin, and $\mathrm{LDH}$, and imaging, including skeletal survey, MRI, CT, and/or PET. One of the two following criteria is required for a diagnosis of PCM:

(1) Clonal bone marrow plasma cells $\geq 10 \%$ or biopsyproven bony or extramedullary plasmacytoma and any one or more of the following signs of end-organ damage that can be attributed to the plasma cell neoplasm:

(i) hypercalcemia, renal insufficiency, anemia, and bone lesions (CRAB criteria).
(2) Clonal bone marrow plasma cells $\geq 10 \%$ or biopsyproven bony or extramedullary plasmacytoma and clonal bone marrow plasma cells $\geq 60 \%$ or involved: uninvolved serum-free light chain ratio $\geq 100$ or more than one focal lesion on MRI [2].

Patients that have $\geq 10 \%$ clonal bone marrow plasma cells but do not show signs of end-organ damage are classified as having a variant of PCM, smouldering (asymptomatic) PCM [2]. With anemia being part of the CRAB criteria for the diagnosis of PCM, it is not surprising that anemia is a common association and complication of PCM, occurring in more than two-thirds of PCM patients [3]. There are multiple proposed mechanisms for the concurrent anemia, including anemia of chronic disease, erythropoietin (EPO) deficiency due to renal problems, and chemotherapy effect upon initiation of treatment [3].

Conversely, polycythemia is an uncommon finding in PCM patients [4]. Polycythemia can be divided into two types, primary and secondary, based on the pathophysiology causing the increased hematocrit. Diseases in which the increased hematocrit is due to a mutation that leads to an 
intrinsic alteration in red blood cells are known as primary polycythemias. Polycythemia vera (PV) is the archetypal primary polycythemia and is almost always caused by a somatic mutation (V617F) in the JAK2 gene that leads to constitutive JAK2 activation and downstream signaling. Primary familial polycythemia (PFP) is caused by an autosomal dominant mutation in the EPO receptor gene [5]. In PFP, the findings are typically isolated erythrocytosis and normal hemoglobin oxygen affinity, with EPO being low to low normal.

Alternatively, secondary polycythemias are caused by increased production of EPO, whether it be the result of chronic hypoxia, due to either diseases such as COPD and chronic sleep apnea or environmental conditions such as living at a high altitude, an EPO-producing neoplastic disease, such as renal cell carcinoma, von Hippel Lindau (VHL) disease, pheochromocytoma, and adrenal adenoma, or a disorder of hypoxia sensing, which are predominantly caused by mutations in VHL or hypoxia-inducible factor (HIF). Chuvash polycythemia, first described in the Chuvash region in Russia, is a rare autosomal recessive congenital polycythemia caused by a $\mathrm{VHL}^{\mathrm{R} 200 \mathrm{~W}}$ germline mutation in the VHL gene. The VHL protein marks the alpha subunits of HIF-1 and HIF-2 for destruction by the proteasome when oxygen levels are normal. In Chuvash polycythemia, the mutation causes impaired recognition of the alpha subunits of HIFs by VHL, leading to impaired degradation of HIF in normoxic conditions, which in turn leads to an aberrant upregulation of the body's hypoxic response and increased EPO production [6].

\section{Case Presentation}

A 48-year-old healthy male presented with a hemoglobin level of $21 \mathrm{mg} / \mathrm{dl}$ and an elevated hematocrit (63\%). The patient had an increased hematocrit (64\%) 12 years ago, leading to clinical suspicion of polycythemia, although the patient was quickly lost to follow-up. At that time, JAK2 mutational testing was negative. At the current presentation, the patient reported fatigue, headache, blurred vision, and excessive sweating. He declined both a history of living at high altitude and smoking. Exogenous EPO use was also excluded. Past medical history includes atrioventricular block requiring pacemaker insertion, hypertension controlled with lisinopril, mild depression managed with citalopram, and erectile dysfunction treated with sildenafil. On physical examination, the patient's vital signs were as follows: blood pressure: 140/100 $\mathrm{mmHg}$; heart rate: 97; respiratory rate: 19 (with excessive redness in the face); BMI: $25.85 \mathrm{~kg} / \mathrm{m}^{2}$; SpO2: 100\%.

\subsection{Labs and Treatment}

WBC: $3.6 \times 10^{9} / \mathrm{L}$

HGB: $19.4 \mathrm{~g} / \mathrm{dL}$

Hct: $63 \%$

MCV: $85 \mathrm{fl} /$ cell

MCH: $26 \mathrm{pg}$

MCHC: $31 \mathrm{~g} / \mathrm{dL}$

\author{
RDW: $25.7 \%$ \\ PLT: $132 \times 10^{9} / \mathrm{L}$ \\ Creatinine: $1.2 \mathrm{mg} / \mathrm{dl}$ (normal range: 0.6-1.2) \\ Carboxyhemoglobin: 1.5\% (normal range: 0-1.4\%) \\ EPO: $687 \mathrm{mU} / \mathrm{ml}$ (normal range: $<29.5$ )
}

CT scans of the chest and abdomen showed no evidence of malignancy, while MRI of the head was negative. Subsequent genetic/biochemical testing ruled out congenital polycythemias.

Due to the polycythemia having no obvious etiology, a bone marrow aspiration and biopsy were performed (Figures 1(a) and 1(b)). The core biopsy showing a markedly decreased myeloid: erythroid ratio with CD71-positive erythroid precursors comprising $\sim 80 \%$ of marrow cellularity (Figure 1(c)) and MPO-positive myeloid cells comprising $<10 \%$ of cells (Figure 1(d)). Surprisingly, the touch prep revealed increased plasma cells (16\%, Figure 2(a)), and CD138 immunohistochemical staining highlighted the increased plasma cells arranged in clusters in the core biopsy ( $\sim 15 \%$ of cellularity, Figure 2(b)). Flow cytometry demonstrated the bone marrow aspirate contained a monotypic lambda-restricted CD38/CD138-positive plasma cell population (Figure 2(c)). Karyotype analysis of the aspirate revealed a normal male 46,XY karyotype, while FISH revealed an IGH/CCND1 rearrangement $(\mathrm{t}(11 ; 14))$, solidifying a diagnosis of plasma cell myeloma. Subsequent serum studies showed increased serum lambda light chains $(41.58 \mathrm{mg} / \mathrm{dl}$; normal range: $0.57-2.63$ $\mathrm{mg} / \mathrm{dl}$ ) and a kappa: lambda ratio of 0.02 . Immunofixation revealed an IgD lambda paraprotein that was too small to quantitate.

2.2. Treatment. The patient was phlebotomized until Hct dropped below 50, which led to the resolution of symptoms. The patient has not been treated for the smouldering PCM.

\section{Discussion}

The combination of polycythemia and PCM is very rare, with most of the cases reported in literature being cases of concurrent PV and PCM [4]. The exclusion of these PV cases leaves a total of six cases in the literature (including this current case of the smouldering variant of PCM which was deemed to be not to be PV due to the absence of the archetypal V617F mutation and elevated EPO levels even though exon 12 mutational studies were not performed), all of which can be characterized as PCM with concurrent polycythemia of undetermined etiology. In three of these cases, the polycythemia significantly preceded the diagnosis of PCM (as in our case), while, in the other two cases, the diseases were diagnosed concurrently [7]. The initial treatment in all of the cases was phlebotomy, with no known treatment for the myeloma in all of the cases in which the polycythemia preceded the myeloma. Interestingly, in the two cases in which the diseases presented concurrently, treatment of the myeloma led to amelioration of both the myeloma and the polycythemia, suggesting an interconnected mechanism $[7,8]$. 


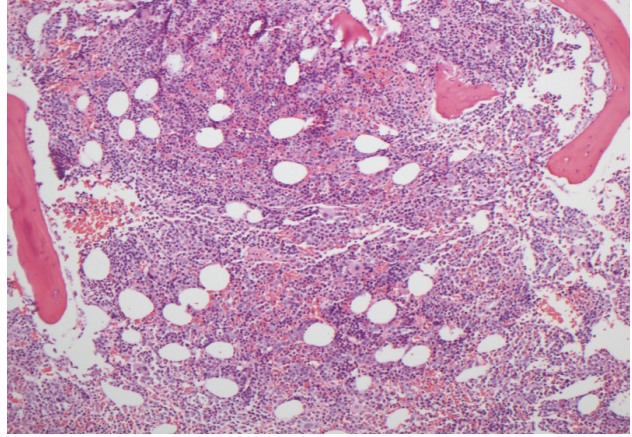

(a)

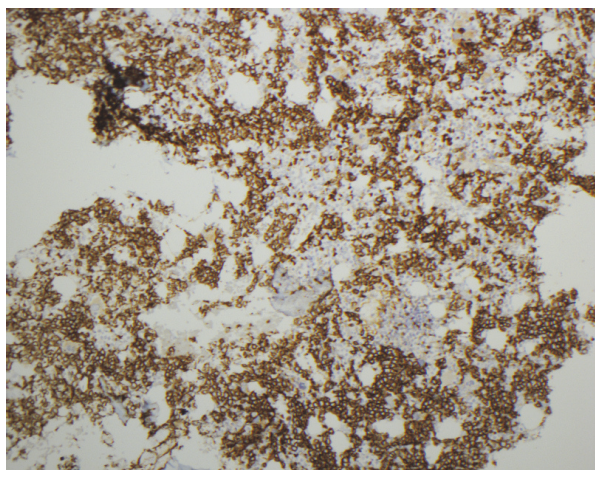

(c)

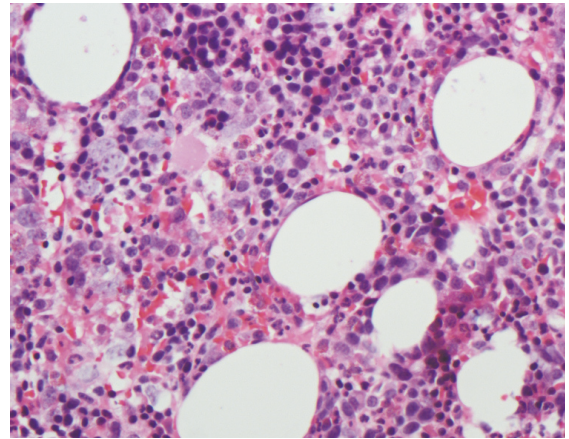

(b)

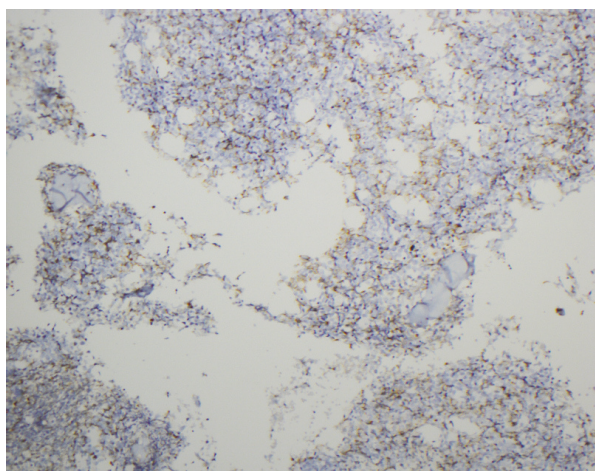

(d)

FiguRE 1: Bone marrow biopsy. (a, b) Representative areas of hematoxylin and eosin-stained sections of the bone marrow biopsy ((a) 10x, (b) 40x). (c) Immunohistochemical (IHC) staining for the erythroid marker CD71 (10x). (d) IHC stain for the myeloid marker MPO (20x).

While the combination of polycythemia and PCM is rare, there are rare syndromes that are characterized by both polycythemia and a monoclonal gammopathy, and these syndromes should always be in the differential in patients with both a monoclonal plasma cell process and polycythemia. Polyneuropathy, organomegaly, endocrinopathy, monoclonal protein, and skin changes (POEMS) syndrome is a rare syndrome with the following diagnostic criteria: major criteria: polyneuropathy, monoclonal plasma disorder, Castleman's disease, sclerotic bone lesions, increased vascular endothelial growth factor; minor criteria: organomegaly, extravascular volume overload, skin changes, endocrinopathy, papilledema, thrombocytosis, and polycythemia [2,9]. A diagnosis requires the fulfillment of at least three major criteria, two of which must be polyradiculoneuropathy and a clonal plasma cell disorder, along with at least one minor criterion [9]. It has been suggested that the manifestations of POEMS are the result of a marked activation of the proinflammatory cytokines IL-1beta, IL-6, and TNF-a along with an associated decrease in the presumptive anti-inflammatory cytokine TGF-beta [10].

Another rare syndrome with erythrocytosis and clonal plasma cells is telangiectasias, elevated EPO and erythrocytosis, monoclonal gammopathy, perinephric fluid collections, and intrapulmonary shunting (TEMPI) syndrome $[2,11]$. The pathogenesis of TEMPI syndrome is still not clear, but it has been hypothesized that the monoclonal protein leads to a paraneoplastic syndrome and increased erythropoietin production, similar to the mechanism seen in solid tumors [11].

In our case, these two syndromes were briefly considered, but as the patient lacked any symptomology other than those due to the polycythemia, criteria were not fulfilled for either of the syndromes. Moreover, the clonal plasma cell process in POEMS usually does not reach the level of PCM (it usually is characterized as a monoclonal gammopathy of undetermined significance (MGUS)) [12], and EPO levels are usually decreased in POEMS [13]. Likewise, the clonal plasma cell process in TEMPI is usually at the level of MGUS [14].

One theory that has been posited in attempt to explain this rare association between PCM and polycythemia is that the deposition of monoclonal light chains causes damage in kidney tubules leading to hypoxia, resulting in increased EPO production. The cases mentioned above in which treatment of the PCM ameliorated the polycythemia are compatible with this theory [7]. This theory seemingly would require the plasma cell malignancy to precede the polycythemia. While at first glance this does not seem congruent with the case we present here since the polycythemia was diagnosed first, it is impossible to know when the patient's asymptomatic monoclonal plasma cell disorder began.

In conclusion, this case highlights that while myeloma is usually associated with anemia, the presence of polycythemia does not rule out plasma cell myeloma, whether it be symptomatic or smouldering. In fact, in rare cases, myeloma can occur concurrently with polycythemia of undetermined 


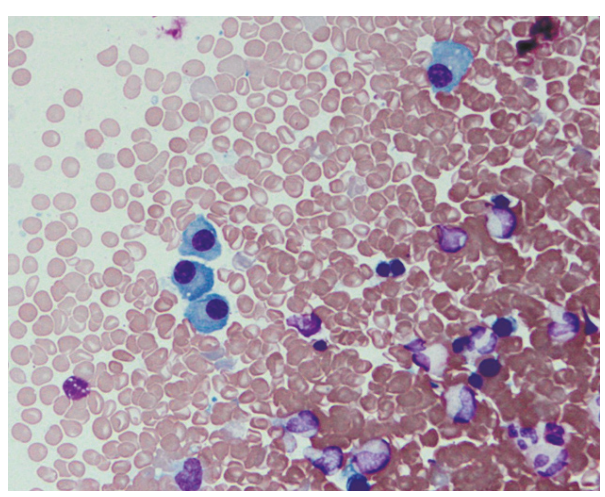

(a)

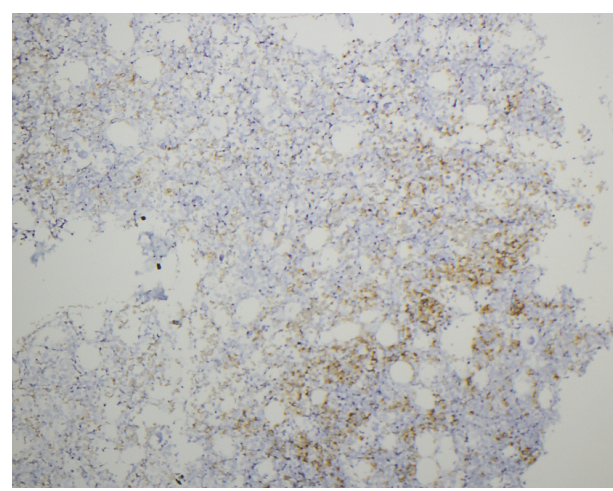

(b)

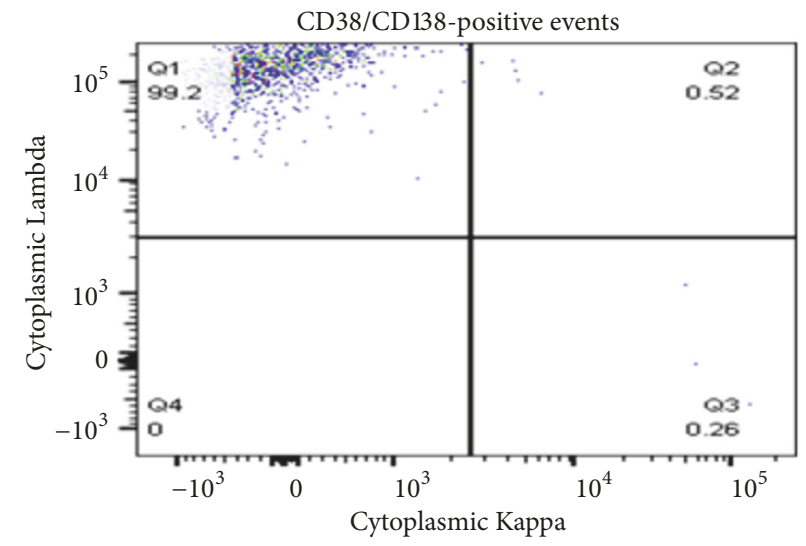

(c)

Figure 2: Evidence of a plasma cell neoplasm. (a) Touch prep of the bone marrow biopsy showing numerous plasma cells (arrow, 50x). (b) IHC staining for the plasma cell marker CD138 (10x). (c) Flow cytometric dot plot of CD38/CD138-positive plasma cells from the bone marrow aspirate showing a monotypic lambda-restricted plasma cell population.

etiology, and the possible interconnection between the two entities is an intriguing area for further studies.

\section{Data Availability}

The data used to support the findings of this study are included within the article.

\section{Disclosure}

A portion of this case was presented at the College of American Pathologists Annual Meeting 2017 in Washington DC and at the Annual Pathology Research Day at University of Rochester Medical Center in June 2018.

\section{Conflicts of Interest}

The authors declare that they have no conflicts of interest.

\section{References}

[1] T. C. Michels and K. E. Petersen, "Multiple myeloma: Diagnosis and treatment," American Family Physician, vol. 95, no. 6, pp. 373-383, 2017.
[2] S. H. Swerdlow, WHO Classification of Tumours of Haematopoietic and Lymphoid Tissues, 2017, WHO classification of tumours of haematopoietic and lymphoid tissues.

[3] H. Ludwig, G. Pohl, and A. Osterborg, "Anemia in multiple myeloma," Clin Adv Hematol Oncol, vol. 2, no. 4, pp. 233-41, 2004.

[4] L. Fink, F. Bauer, and J. J. Perry, "Coincidental polycythemia vera and multiple myeloma: Case report and review," American Journal of Hematology, vol. 44, no. 3, pp. 196-200, 1993.

[5] S. E. Langabeer, "The JAK2 V617F mutation in plasma cell neoplasms with co-existing erythrocytosis," Journal of Clinical and Diagnostic Research, vol. 9, no. 12, p. EL01, 2015.

[6] G. Y. Miasnikova, A. I. Sergueeva, M. Nouraie et al., "The heterozygote advantage of the chuvash polycythemia VHL R200W mutation may be protection against anemia," Haematologica, vol. 96, no. 9, pp. 1371-1374, 2011.

[7] E. J. Hutchison, J. A. Taverna, Q. Yu, and A. M. Yeager, "Polycythaemia: an unusual presentation of multiple myeloma," BMJ Case Reports, vol. 2016, 2016.

[8] S.-G. Lee, G. Lim, S. Y. Cho et al., "JAK2 mutation-negative secondary erythrocytosis in smoldering plasma cell myeloma: A case study and review of the literature," Acta Haematologica, vol. 126, no. 3, pp. 169-171, 2011. 
[9] A. Dispenzieri, "POEMS syndrome: 2017 Update on diagnosis, risk stratification, and management," American Journal of Hematology, vol. 92, no. 8, pp. 814-829, 2017.

[10] R. K. Gherardi, L. Bélec, M. Soubrier et al., "Overproduction of proinflammatory cytokines imbalanced by their antagonists in POEMS syndrome," Blood, vol. 87, no. 4, pp. 1458-1465, 1996.

[11] F. G. Rosado, J. L. Oliveira, A. R. Sohani et al., "Bone marrow findings of the newly described TEMPI syndrome: When erythrocytosis and plasma cell dyscrasia coexist," Modern Pathology, vol. 28, no. 3, pp. 367-372, 2015.

[12] A. Dispenzieri, R. A. Kyle, M. Q. Lacy et al., "POEMS syndrome: Definitions and long-term outcome," Blood, vol. 101, no. 7, pp. 2496-2506, 2003.

[13] M. Scarlato, S. C. Previtali, M. Carpo et al., "Polyneuropathy in POEMS syndrome: role of angiogenic factors in the pathogenesis," Brain, vol. 128, no. 8, pp. 1911-1920, 2005.

[14] D. B. Sykes, W. Schroyens, and C. O'Connell, “The TEMPI syndrome - A novel multisystem disease," The New England Journal of Medicine, vol. 365, no. 5, pp. 475-477, 2011. 


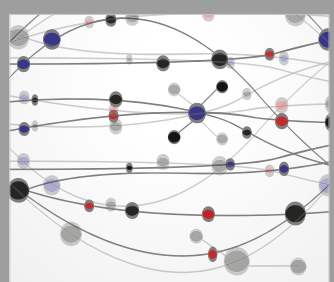

The Scientific World Journal
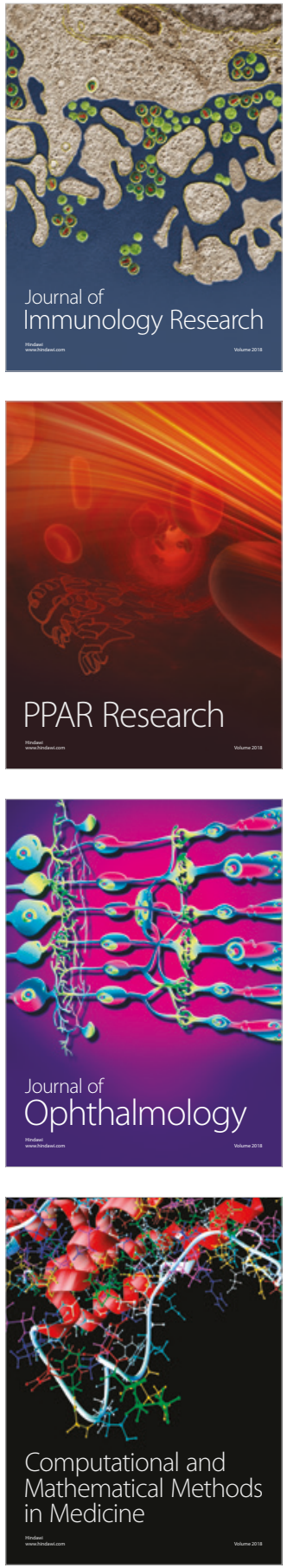

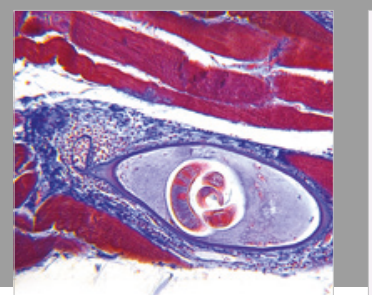

Gastroenterology Research and Practice

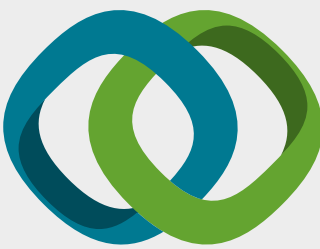

\section{Hindawi}

Submit your manuscripts at

www.hindawi.com
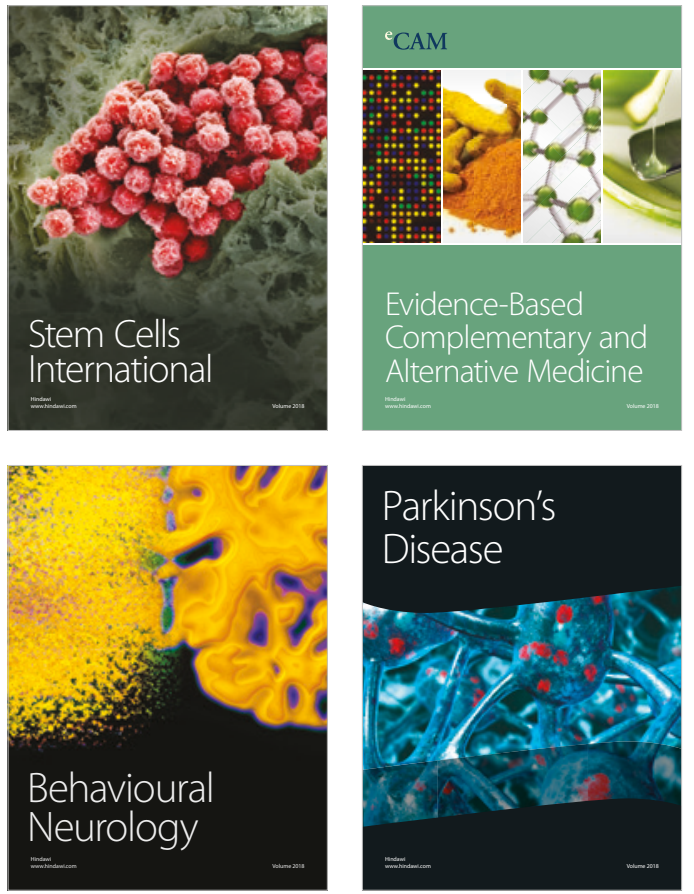

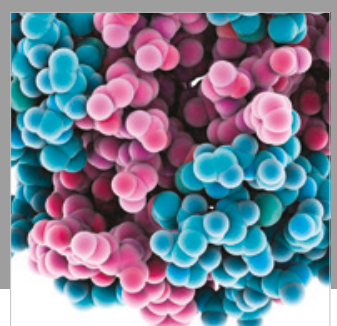

ournal of

Diabetes Research

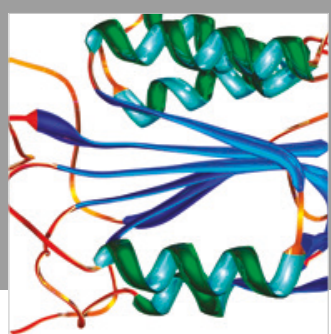

Disease Markers
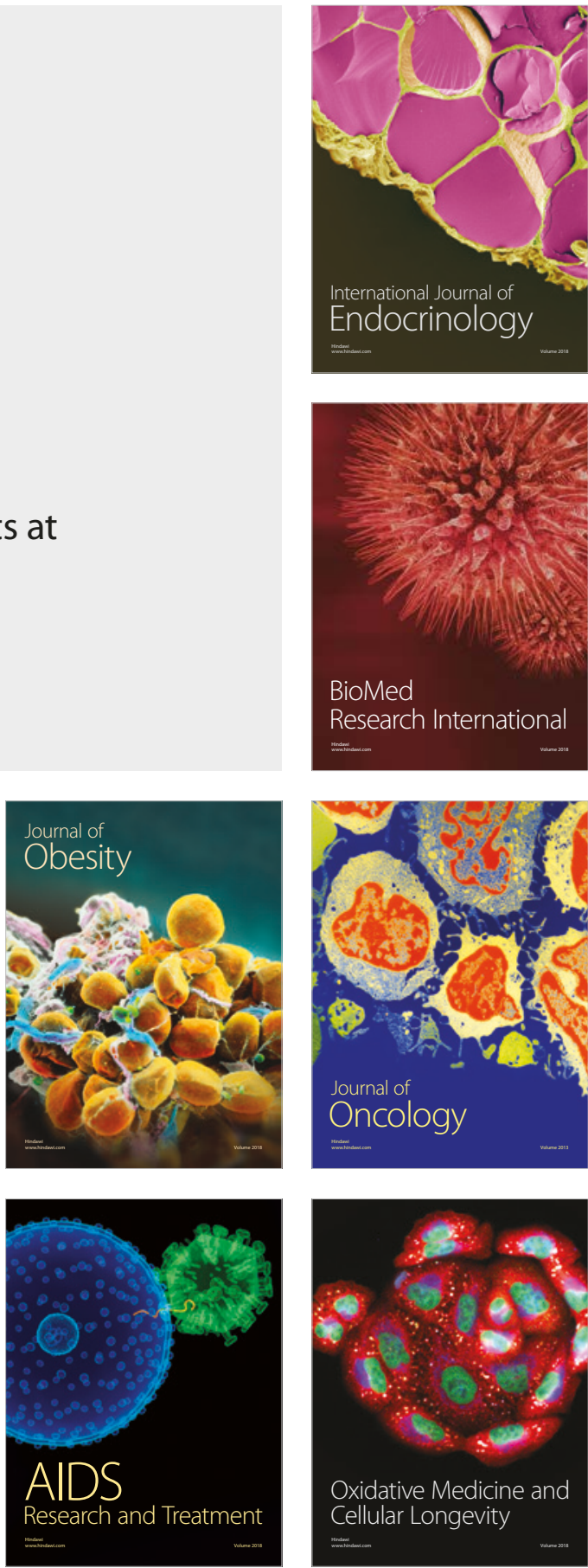